\title{
Small molecule sensitization to TRAIL is mediated via nuclear localization, phosphorylation and inhibition of chaperone activity of Hsp27
}

\author{
G Mellier $^{1,2}$, D Liu ${ }^{1,3}$, G Bellot ${ }^{1}$, A Lisa Holme ${ }^{4,6}$ and S Pervaiz ${ }^{\star, 1,2,3,4,5}$
}

The small chaperone protein Hsp27 confers resistance to apoptosis, and therefore is an attractive anticancer drug target. We report here a novel mechanism underlying the tumor necrosis factor-related apoptosis-inducing ligand (TRAIL) sensitizing activity of the small molecule LY303511, an inactive analog of the phosphoinositide 3-kinase inhibitor inhibitor LY294002, in HeLa cells that are refractory to TRAIL-induced apoptosis. On the basis of the fact that LY303511 is derived from LY294002, itself derived from quercetin, and earlier findings indicating that quercetin and LY294002 affected Hsp27 expression, we investigated whether LY303511 sensitized cancer cells to TRAIL via a conserved inhibitory effect on Hsp27. We provide evidence that upon treatment with LY303511, Hsp27 is progressively sequestered in the nucleus, thus reducing its protective effect in the cytosol during the apoptotic process. LY303511-induced nuclear translocation of Hsp27 is linked to its sustained phosphorylation via activation of p38 kinase and MAPKAP kinase 2 and the inhibition of PP2A. Furthermore, Hsp27 phosphorylation leads to the subsequent dissociation of its large oligomers and a decrease in its chaperone activity, thereby further compromising the death inhibitory activity of Hsp27. Furthermore, genetic manipulation of Hsp27 expression significantly affected the TRAIL sensitizing activity of LY303511, which corroborated the Hsp27 targeting activity of LY303511. Taken together, these data indicate a novel mechanism of small molecule sensitization to TRAIL through targeting of Hsp27 functions, rather than its overall expression, leading to decreased cellular protection, which could have therapeutic implications for overcoming chemotherapy resistance in tumor cells. Cell Death and Disease (2013) 4, e890; doi:10.1038/cddis.2013.413; published online 31 October 2013

Subject Category: Cancer

Since its discovery almost two decades ago, tumor necrosis factor (TNF)-related apoptosis-inducing ligand (TRAIL) has garnered heightened interest due to its ability to selectively induce apoptosis in tumor cells, whereas eliciting minimal toxicity to normal cells. ${ }^{1-4}$ However, there is sufficient experimental evidence that a variety of human tumors are intrinsically resistant to TRAIL-induced apoptosis or could rapidly acquire a resistant phenotype. ${ }^{5-7}$ Nevertheless, the promise of a selective therapeutic agent as well as the growing understanding of TRAIL signaling, in particular the focused attention on the mechanisms responsible for resistance, have triggered an intense search for novel compounds that are able to restore tumor cell sensitivity to TRAIL. To that end, a large number of small molecules have shown promising results, some of which are already used as mono-therapeutic agents in the clinic (e.g., fluorouracil, etoposide and cisplatin). ${ }^{8-10}$ Previously, we reported that LY303511 (LY30), an inactive analog of the phosphoinositide 3-kinase inhibitor (PI3K) LY294002 (LY29), could strongly enhance tumor cells sensitivity to TRAILmediated apoptosis in a PI3K-independent manner. Although, these data indicated involvement of reactive oxygen species (ROS)-mediated mitogen-activated protein kinases (MAPKs) activation, amplification of the apical TRAIL signal and robust activation of the intrinsic apoptotic pathway, ${ }^{11,12}$ our recent work has focused on identifying underlying resistance mechanisms targeted by L30.

Hsp27 is a member of the small heat-shock proteins (sHsps). Hsp27 is a stress-activated chaperone protein that displays an abnormal expression in numerous tumor cells and provides them with an enhanced protection against druginduced cell death. ${ }^{13,14}$ Hsp27 has an important role in inhibiting both extrinsic and intrinsic cell death pathways by preventing the translocation of the truncated form of BID to the mitochondria. ${ }^{15}$ It also interacts with cytochrome $\mathrm{c}$ after its release from the mitochondria ${ }^{16}$ and can disrupt caspase- 3 activation. ${ }^{17}$ In addition, Hsp27 possesses an ATP-independent chaperone-like activity due to the ability of its large oligomers to form reservoirs of misfolded proteins during cellular stresses, thereby attenuating the irreversible and toxic aggregation of denatured proteins. ${ }^{18}$

\footnotetext{
${ }^{1}$ ROS, Apoptosis and Cancer Biology Laboratory, Department of Physiology, Yong Loo Lin School of Medicine, National University of Singapore, Singapore, Singapore; ${ }^{2}$ Cancer and Stem Cell Biology Program, Duke-NUS Graduate Medical School, Singapore, Singapore; ${ }^{3}$ NUS Graduate School for Integrative Sciences and Engineering, National University of Singapore, Singapore, Singapore; ${ }^{4}$ Cancer Science Institute, National University of Singapore, Singapore, Singapore and ${ }^{5}$ Singapore-MIT Alliance, Singapore, Singapore

*Corresponding author: S Pervaiz, ROS, Apoptosis and Cancer Biology Laboratory, Department of Physiology, Yong Loo Lin School of Medicine, National University of Singapore, Singapore 117597, Singapore. Tel: 65 96614732; Fax: 65 67788161; E-mail: phssp@ nus.edu.sg

${ }^{6}$ Current address: Department of Medical Microbiology \& Immunology, University of Alberta, Edmonton, AB, Canada T6G 2E1

Keywords: drug resistance; apoptosis; TRAIL; LY303511; Hsp27

Abbreviations: (s)Hsp, (small) heat-shock proteins; LY29, LY294002; LY30, LY303511; MK2, MAPKAPK2; PI3K, phosphoinositide 3-kinase; PP2A, Protein Phosphatase 2 Alpha; ROS, reactive oxygen species; TRAIL, TNF-related apoptosis-inducing ligand

Received 17.4.13; revised 19.9.13; accepted 20.9.13; Edited by T Brunner
} 
In this study, we evaluated the effect of LY30 with respect to Hsp27 survival functions based on our previous work hinting at a pleiotropic role of this small molecule. We show that LY30, independently of TRAIL, induced sustained Hsp27 phosphorylation via activation of the $\mathrm{p38} / \mathrm{MK} 2$ pathway and inhibition of PP2A, leading to Hsp27 de-oligomerization and nuclear sequestration as well as impairment of its chaperone activity. These data shed light on a novel mechanism, independent of TRAIL apical signaling, by which the small molecule LY30 restores or enhances cancer cells' sensitivity to TRAIL treatment.

\section{Results}

LY30 restores sensitivity to TRAIL-induced cell death. First, the variant of human cervical cancer HeLa cell line used in this study was significantly refractory to long-term treatment with TRAIL (for up to $72 \mathrm{~h}$ ), with only $250 \mathrm{ng} / \mathrm{ml}$ of TRAIL for $72 \mathrm{~h}$ eliciting a modest effect on cell viability (Figure 1a). However, as seen in Figure 1b, addition of LY30 restored HeLa sensitivity to TRAIL in a synergistic manner, corroborating our earlier findings that LY30 sensitized human tumor cells to TRAIL-induced cell death. ${ }^{11,12}$ The synergism between LY30 and TRAIL was assessed by calculating the combination index $(\mathrm{Cl})$ using the Chou-Talalay method $(\mathrm{Cl}=0.030$ for $25 \mu \mathrm{M}$ of $\mathrm{LY} 30$ and $20 \mathrm{ng} / \mathrm{ml}$ of TRAIL) (Figure 1b). ${ }^{19}$ The same concentrations were used throughout the rest of the study. Dose-response curves for TRAIL and LY30 were also established by evaluating the effect of increasing doses of TRAIL $(0,10$ and $20 \mathrm{ng} / \mathrm{ml})$ and LY30 $(0-6.25 \mu \mathrm{M})$ on cell viability after $16 \mathrm{~h}$ (Figure 1c). Interestingly, different doses of TRAIL did not yield significantly different results, whereas increasing the dose of LY30 significantly decreased cell viability in a dose-dependent manner up to $3.125 \mu \mathrm{M}$ when it reached a plateau. This result shows that the sensitization is highly dependent on LY30 concentration at lower doses, whereas lower doses of TRAIL are sufficient. As in our earlier reports, the observed cell death was apoptotic as seen by the dependency on caspases activation and engagement of the mitochondrial cell death pathway (Supplementary Figure 1). Interestingly, the observed sensitization was enhanced in a dosedependent manner as a function of TRAIL concentration. In HeLa cells treated with $25 \mu \mathrm{M}$ LY30 and $20 \mathrm{ng} / \mathrm{ml}$ TRAIL a
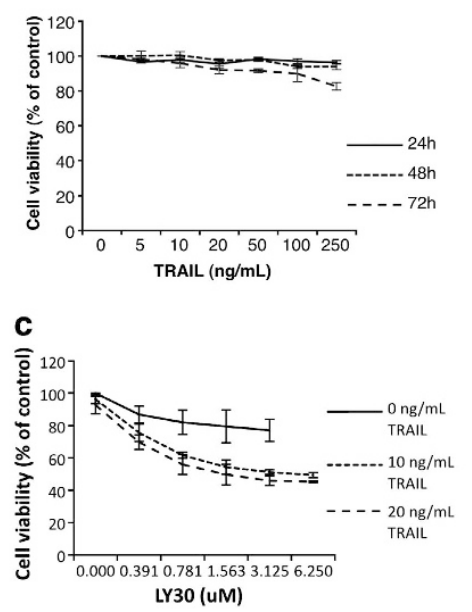

b
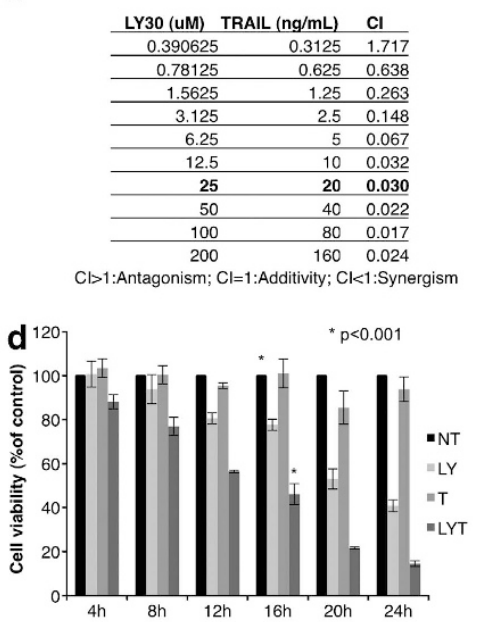

e

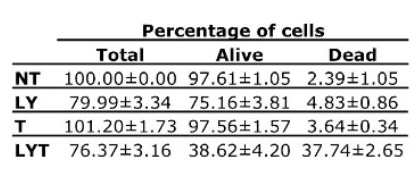

f

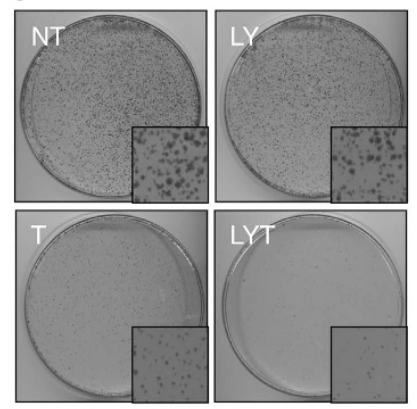

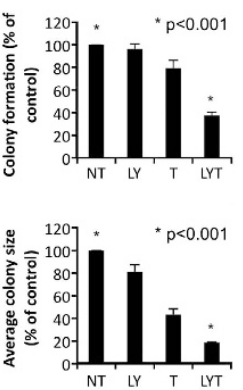

Figure 1 LY30 restores sensitivity to TRAIL-mediated cell death. (a) Crystal violet viability assay of HeLa cells treated with treated with TRAIL alone (0-250 $\mathrm{ng} / \mathrm{ml})$ for 24 , 48 or $72 \mathrm{~h}$. (b) Cl of LY30 and TRAIL calculated with the Chou-Talalay method. (c) Crystal violet viability assay of HeLa cells treated with TRAIL $(0,10 \mathrm{and} 20 \mathrm{ng} / \mathrm{ml})$ for $16 \mathrm{~h}$ with or without $1 \mathrm{~h}$ preincubation with various doses of LY30 $(0-6.125 \mu \mathrm{M})$. (d) Crystal violet viability assay of HeLa cells treated with $25 \mu \mathrm{M}$ of LY30 and $20 \mathrm{ng} / \mathrm{ml}$ of TRAIL for various durations. (e) Trypan blue exclusion assay for HeLa cells treated with $20 \mathrm{ng} / \mathrm{ml}$ of TRAIL for $16 \mathrm{~h}$ with or without preincubation with $25 \mu \mathrm{M} \mathrm{LY} 30$. (f) Colony-forming assay for HeLa cells treated with $20 \mathrm{ng} / \mathrm{ml}$ of TRAlL for $6 \mathrm{~h}$ with or without preincubation with $25 \mu \mathrm{M} \mathrm{LY} 30$ before re-seeding. Colony count and colony size measurements are also provided. Data shown are the mean \pm S.D. of three independent experiments. NT: not treated, LY: LY30, T: TRAIL, LYT: LY30 + TRAIL 
alone or in combination, viability was decreased in a timedependent manner (Figure 1d). Of note, at 16 h of treatment, the lower viability readings seen upon treatment with LY30 alone could be attributed to a decrease in cell number rather than cell death (Figure 1e). In addition to the enhanced sensitization of HeLa cells to TRAIL by LY30, the two drugs in combination inhibited the colony-forming ability of the tumor cells, whereas neither drug used alone had any effect on tumor colony-forming ability, although TRAIL alone was able to reduce the size of the colonies (Figure 1f).

Hsp27 silencing/overexpression directly affects cells sensitivity/resistance to TRAIL. Several studies have previously shown that both quercetin and LY29 (which is derived from quercetin) (Supplementary Figure 2) have an effect on the expression and/or induction of the survival protein Hsp27. ${ }^{20-23}$ As LY30 is derived from LY29, we were interested to see whether artificial modulation of Hsp27 protein expression (Figure 2a) could affect the outcome of TRAIL and/or LY30 treatment. Overexpression of Hsp27 significantly rescued cells treated with LY30 and TRAIL $(\geq 20 \mathrm{ng} / \mathrm{ml}$ ) for $16 \mathrm{~h}$ as compared with control cells (Figures $2 \mathrm{~b}$ and $\mathrm{c}$ ). Inversely, knockdown of Hsp27 expression by siRNAs sensitized cells exposed to the combined treatment, with a $20 \%$ difference for all doses of TRAIL used as compared with mock transfected cells (Figure 2d). Although silencing Hsp27 did not sensitize HeLa cells when TRAIL was used alone, even at the highest concentration
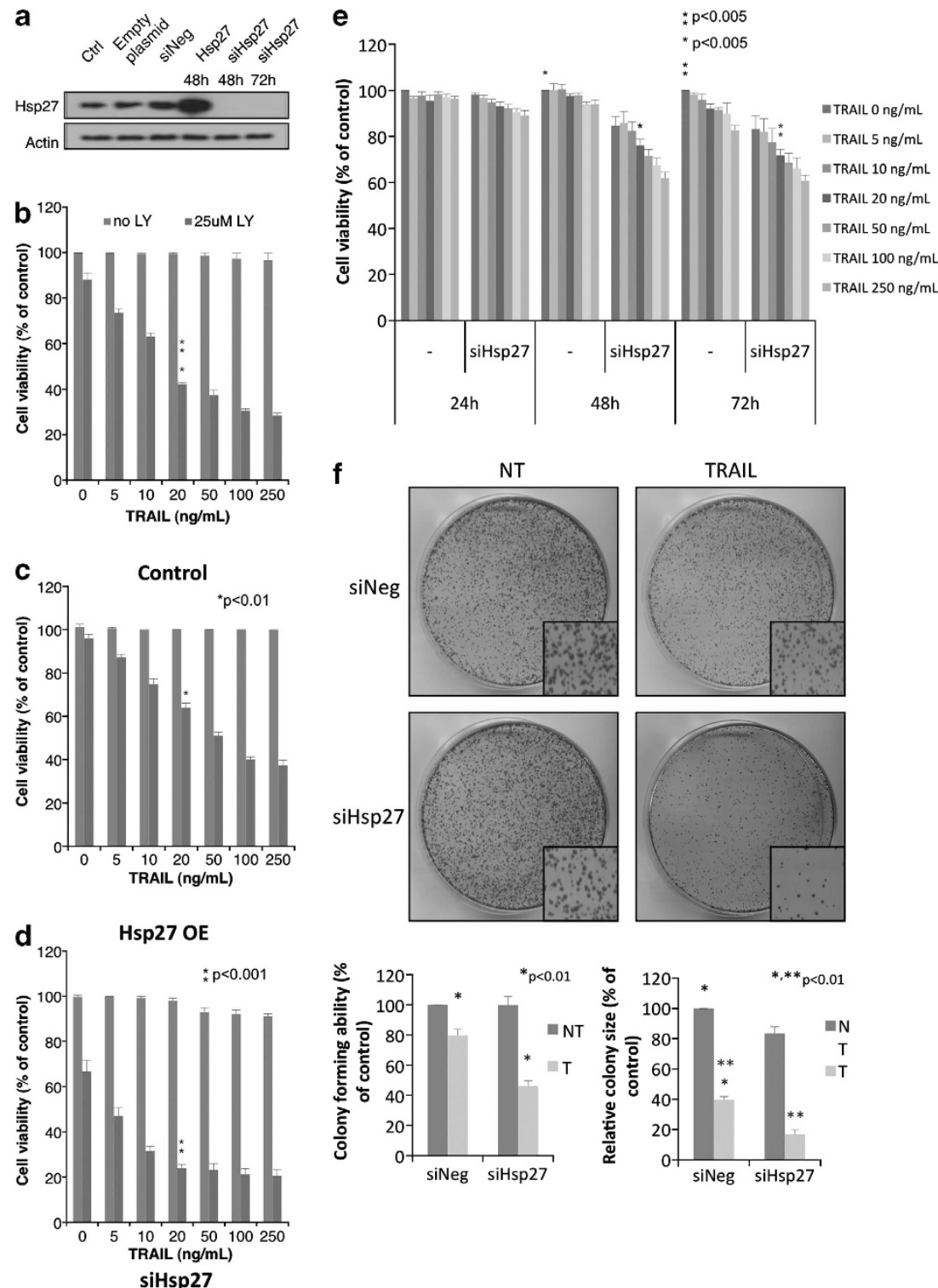

Figure 2 Effect of Hsp27 expression modulation. (a) Western blot analysis of non transfected Hela cells, cells transiently overexpressing Hsp27 or cells where Hsp27 has been knocked down. The indicated durations represent the time after transfection. (b) Crystal violet viability assay of HeLa cells after $16 \mathrm{~h}$ treatment with TRAIL (0-250 ng/ml) in the presence or absence of $25 \mu \mathrm{M}$ LY30 in non transfected cells, (c) in cells transiently overexpressing Hsp27 or (d) in cells where Hsp27 has been knocked down. (e) Crystal violet viability assay of HeLa cells transfected with negative siRNA or specific for Hsp27 and treated with TRAlL (0-250 ng/ml) for 24, 48 and $72 \mathrm{~h}$. (f) Colony formation assay in cells transfected with negative siRNA or specific for Hsp27 and treated with $20 \mathrm{ng} / \mathrm{ml}$ TRAIL. Colony count and colony size are provided 
(250 ng/ml), long-term survival and colony-forming experiments showed a decreased cell viability/colony-forming ability (Figures $2 e$ and f). The decrease in cell viability was dependent on the dose of TRAIL (Figure 2e). In addition, the decrease in colony-forming ability was accompanied by a significant decrease in colony size as compared with the control.

These results suggest that Hsp27 has a role in LY30mediated sensitization of HeLa cells to TRAIL-induced apoptosis as well as a role in TRAIL resistance in our cell system, although the involvement of Hsp27 in TRAIL resistance is only visible in medium- and long-term survival assays.

Hsp27 localization, but not its expression, changes upon exposure to LY30. After determining that Hsp27 had a part in TRAIL resistance and could also negatively influence LY30 resensitization, we decided to investigate whether LY30 also had an effect on Hsp27 expression as previously reported by other groups for LY29 and quercetin. $^{20-23}$ Results show that LY30 treatment, alone or in combination with TRAIL, induced a decrease in Hsp27 protein levels in cleared RIPA lysates as evidenced by western blot analysis (Figure 3a). However, no change could be observed in Hsp70 or Hsp90 protein levels, suggesting that LY30 had an effect primarily on Hsp27. We further investigated this reduction in Hsp27 protein level upon LY30 treatment by examining Hsp27 mRNA expression as well as the effect of the inhibition of proteasomal degradation. Interestingly, no decrease in Hsp27 total RNA could be detected at any of the time points tested (Figure 3b), indicating that LY30-mediated decrease in Hsp27 expression was not a function of its transcriptional regulation. Surprisingly, pretreatment with the proteasomal inhibitor lactacystin had no effect on Hsp27 decrease upon LY30 treatment (Figure 3c). Taken together, these results indicate that the decrease in Hsp27 protein level observed upon LY30 treatment was neither due to transcriptional regulation of Hsp27 nor due to an increase in Hsp27 protein degradation.

Interestingly, Hsp27 is known to translocate to the nucleus upon certain cellular stresses. We suspected that the cell lysis method used (i.e., lysis with RIPA buffer followed by clearing with centrifugation) did not provide complete lysis of the cells and nuclei and could lead to the loss of a significant fraction of total Hsp27 protein during the clearing step. Indeed, analysis of the nuclei-enriched pellet obtained after sample clearing showed an increase in the Hsp27 protein level mirroring the decrease observed in the supernatant (Figure 3d). The lack of such changes in whole-cell lysates or the supernatant and pellet fractions of sonicated samples (Figures $3 e$ and $f$ ) confirmed the incomplete lysis hypothesis and indicated that LY30 had no effect on Hsp27 expression but instead induced its translocation to a nuclei-enriched pellet fraction (Figures $3 e$ and $\mathrm{f}$ ).

Sustained Hsp27 nuclear translocation during LY30 treatment. In order to ascertain that Hsp27 was translocated to the nucleus, we set out to monitor the presence of Hsp27 in cytosolic and nuclear fractions obtained by using classical cellular fractionation methods. As seen in figure $4 a$, Hsp27 protein level decreases in the cytosolic fraction after treatment with LY30, alone or in combination with TRAIL, but concomitantly increases in the nuclei-enriched fraction as early as $4 \mathrm{~h}$, hence confirming previous results. A gentler
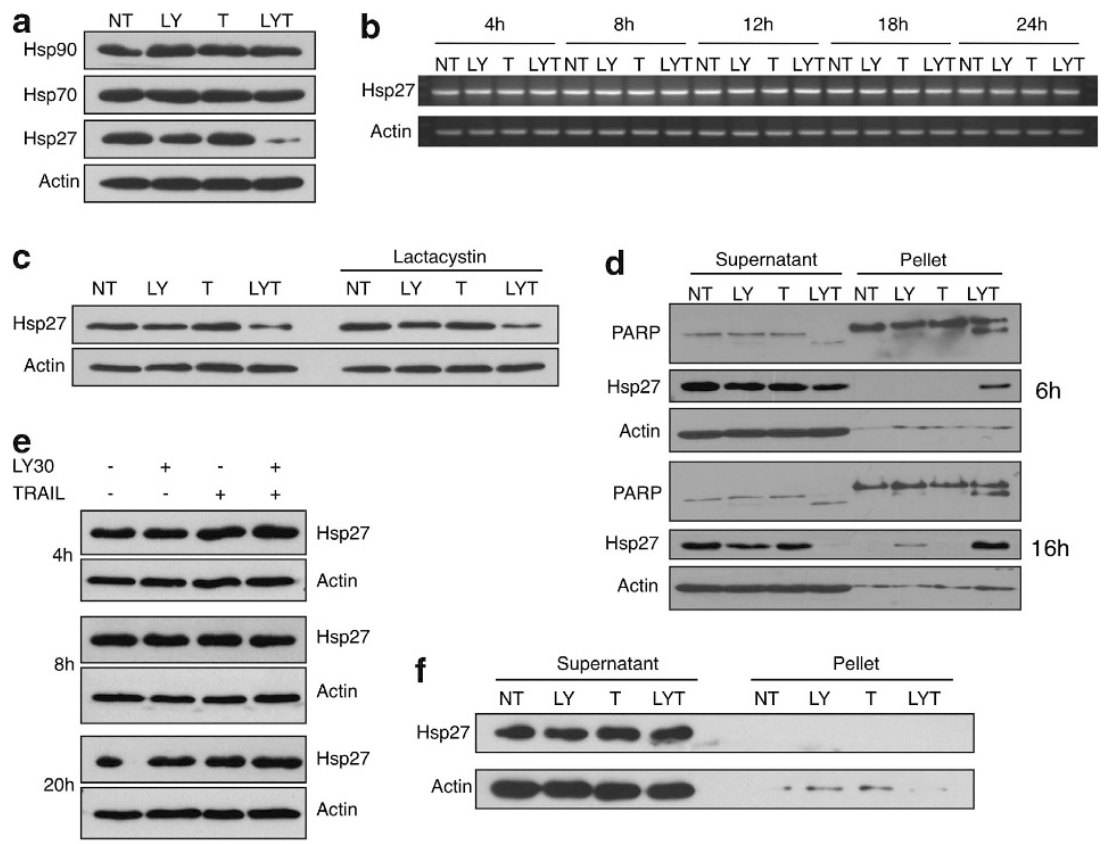

Figure 3 Changes in Hsp27 localization but not expression upon exposure to LY30. (a) Western blot analyses of Hsp27, Hsp70 and Hsp90 after $20 \mathrm{~h}$ of treatment with LY30. (b) Total mRNA levels of Hsp27 in HeLa cells after treatment for different durations. (c) Western blot analysis of Hsp27 levels after $16 \mathrm{~h}$ of treatment in the presence or absence of $10 \mu \mathrm{M}$ lactacystin ( $2 \mathrm{~h}$ preincubation). (d) Western blot analysis of $\mathrm{Hsp} 27$ in the pellet and supernatant fractions of cleared (centrifuged) cell lysates after 6 or $16 \mathrm{~h}$ of treatment, (e) in whole-cell lysates after 4,8 or $20 \mathrm{~h}$ of treatment or $(\mathrm{f})$ in the pellet and supernatant fractions of sonicated cell lysates after $16 \mathrm{~h}$ of treatment 


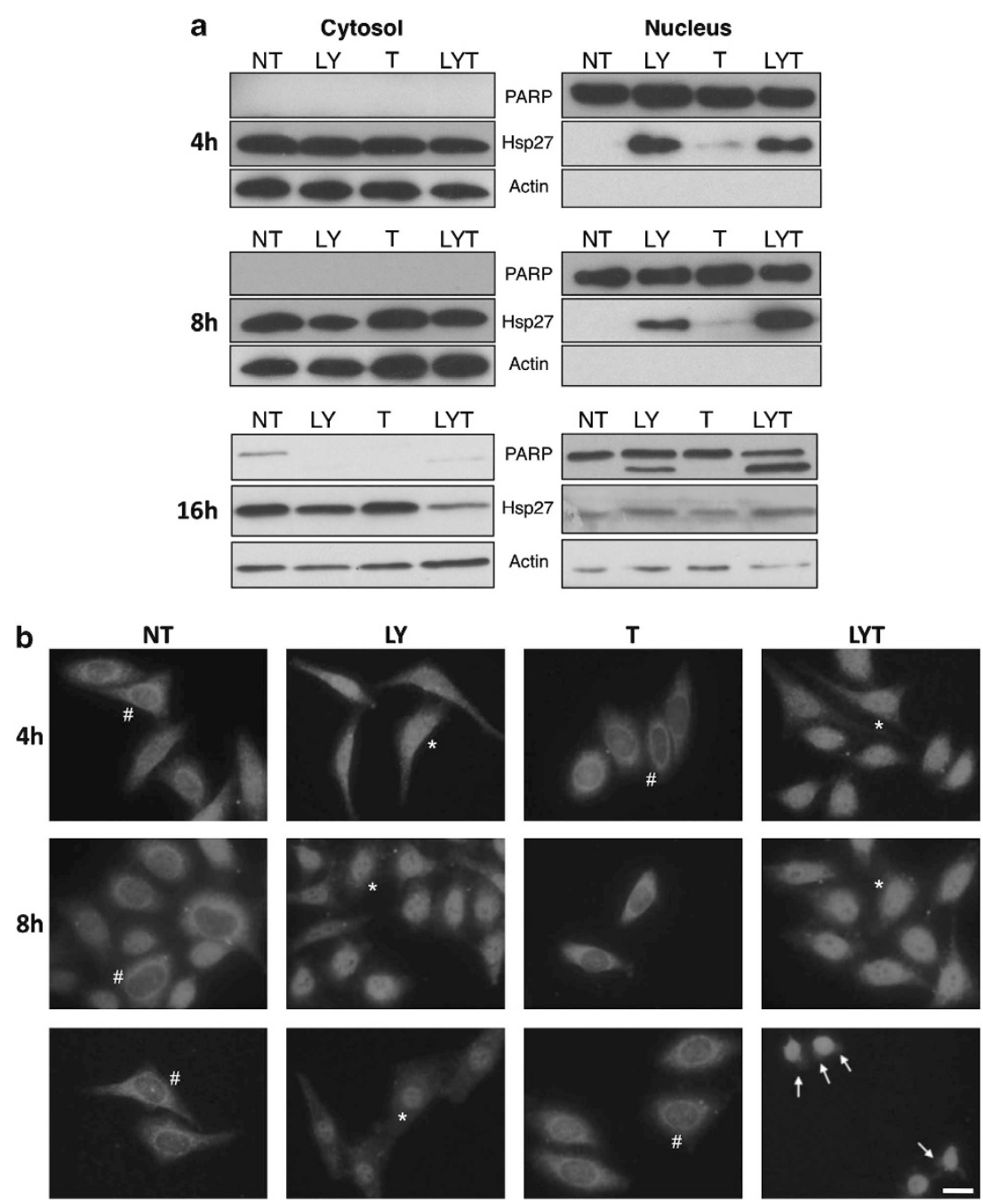

Figure $4 \mathrm{Hsp27}$ translocates to the nucleus. (a) Western blot analysis of Hsp27 in the nuclear and cytosolic fractions of HeLa cells following 4,8 or 16 h exposure to $25 \mu \mathrm{M}$ LY30 and/or $20 \mathrm{ng} / \mathrm{ml}$ TRAlL. (b) Immunofluorescence analysis of Hsp27 cellular localization (red: Hsp27). \# denotes a representative cytoplasmic localization of Hsp27, whereas * denotes a representative nuclear localization of Hsp27. Scale bar: $10 \mu \mathrm{m}$

nuclear fractionation method was used to monitor Hsp27 localization at the $16 \mathrm{~h}$ time point, as the previous method was not suited when nuclei are damaged. We observed the same trend as for earlier time points. These results were confirmed by immunofluorescence analysis at 4,8 and $16 \mathrm{~h}$ showing a time-dependent increase in nuclear staining in cells treated with LY30 confirming that Hsp27 does indeed specifically translocate to the nucleus after treatment with LY30 (Figure $4 \mathrm{~b}$ and Supplementary Figure 3). It is important to note that Hsp27 translocation is gradual and sustained (up to $16 \mathrm{~h}$ ), which is in sharp contrast with the rapid and transient nuclear translocation observed during a heat shock (Supplementary Figure 4).

Hsp27 phosphorylation is sustained through activation of p38/MK2 and inhibition of PP2A. After confirming that LY30 could induce Hsp27 translocation to the nucleus, we set out to investigate the mechanisms involved in this translocation. Even though the precise mechanism by which Hsp27 enters or leaves the nucleus is still unknown, it is commonly accepted that it occurs via passive transport through the nuclear pores. Therefore, the size of Hsp27 oligomers has to be small enough in order to permit entry into the nucleus. As Hsp27 oligomerization is dependent on its phosphorylation, we set out to analyze the phosphorylation status of Hsp27 upon treatment with LY30 and TRAIL. A marked increase in phosphorylation of Hsp27 on Ser-82 residue was observed as early as $15 \mathrm{~min}$ after exposure to LY30 (Figure 5a) and was sustained for up to $16 \mathrm{~h}$ (Supplementary Figure 5, Figures 5b-d). Of note, TRAIL alone did not induce Hsp27 phosphorylation (Figure 5b).

Hsp27 is phosphorylated by MAPKAPK2 (MK2), which is itself phosphorylated by p38 MAPK. Interestingly, both p38 and MK2 were activated upon LY30 treatment and pretreatment with SB203580 (a specific p38 inhibitor) abrogated the phosphorylation of p38, MK2 and Hsp27 (Figures $5 \mathrm{a}-\mathrm{c}$ ). Hsp27 phosphorylation can also be regulated by PP2A. Indeed, pretreatment with okadaic acid resulted in increased phosphorylation of Hsp27 (Figure 5d), which suggested an effect of LY30 on PP2A. Indeed, 
a
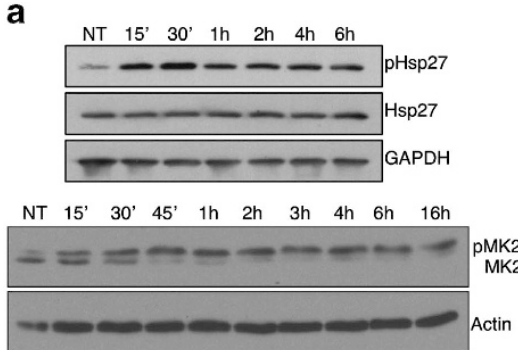

C

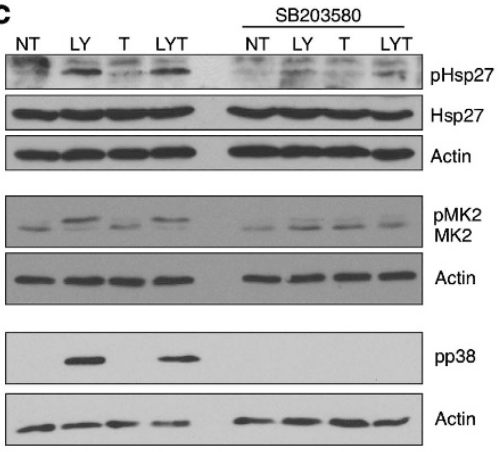

b
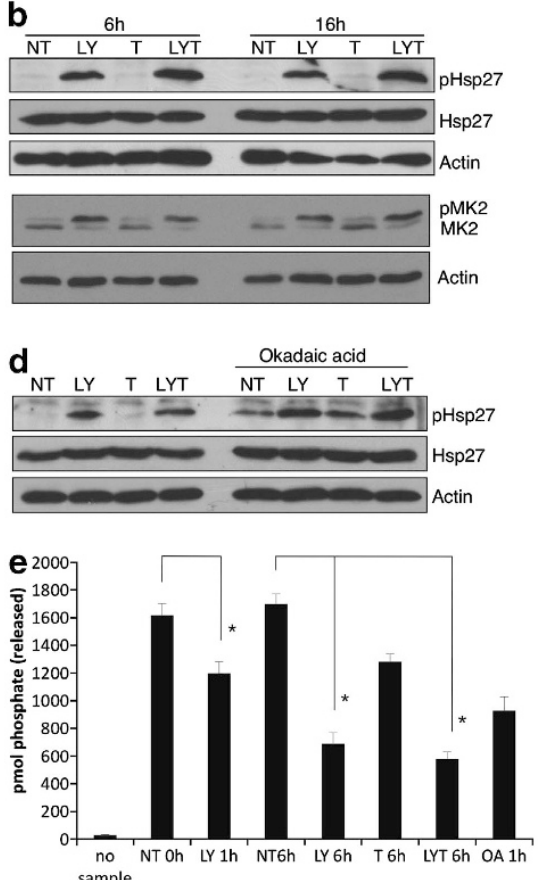

Figure 5 Sustained Hsp27 phosphorylation. (a) Western blot analysis of total Hsp27, phospho-Hsp27 and MK2 after treatment with $25 \mu \mathrm{M} L Y 30$ for the indicated times or (b) treated for 6 or $16 \mathrm{~h}$ with $20 \mathrm{ng} / \mathrm{ml}$ TRAIL with or without preincubation with $25 \mu \mathrm{M} \mathrm{LY30.} \mathrm{(c)} \mathrm{Western} \mathrm{blot} \mathrm{analysis} \mathrm{of} \mathrm{total} \mathrm{Hsp27,} \mathrm{phospho-Hsp27,} \mathrm{phospho-p38,} \mathrm{MK2} \mathrm{and}$ phospho-MK2 after $16 \mathrm{~h}$ in the presence or absence of $10 \mu \mathrm{M} \mathrm{SB203580.} \mathrm{(d)} \mathrm{Western} \mathrm{blot} \mathrm{analysis} \mathrm{of} \mathrm{total} \mathrm{Hsp27,} \mathrm{phospho-Hsp27} \mathrm{after} 16 \mathrm{~h}$ treatment in the presence or absence of $10 \mu \mathrm{M}$ okadaic acid. (e) PP2A phosphatase activity following the indicated treatments (OA: okadaic acid) ${ }^{*} P<0.01$

a significant inhibition of PP2A activity could be observed upon treatment with LY30 alone as early as $1 \mathrm{~h}$ (Figure 5e). Interestingly, an even stronger inhibition was observed after $6 \mathrm{~h}$ in samples treated with LY30, whereas no additive effect could be observed with the combined treatment. Taken together, these results indicate that LY30 is able to induce Hsp27 phosphorylation by activating p38 MAKP as well as inhibiting PP2A.

LY30 induces a shift in Hsp27 oligomeric size toward small oligomers. Having established the ability of LY30 to modulate Hsp27 phosphorylation status, we set out to investigate the subsequent effect on Hsp27 oligomerization. To that end, we performed glycerol gradient ultracentrifugation. In untreated cells, Hsp27 sedimented as complexes of heterogeneous sizes distributed between the top of the gradient (monomers, dimers and low-molecular size oligomers) and fractions corresponding to a high-molecular masses (Figures $6 \mathrm{a}$ and $\mathrm{b}$ ). Interestingly, $1 \mathrm{~h}$ treatment with LY30 resulted in a drastic change in the size distribution of Hsp27 oligomers with a shift toward the lower molecular size oligomers. A longer exposure to LY30, alone or in combination with TRAIL, led to a similar shift toward small oligomers. To confirm these results, glutaraldehyde cross-linking experiments were performed (Figure 6c). Hsp27 cross-linking was markedly reduced in LY30-treated samples, in particular the higher size cross-linked products. Moreover, treatment with okadaic acid, which is able to increase Hsp27 phosphorylation, induced a comparable shift. Inversely, pretreatment with the p38 MAPK inhibitor SB203580 abrogated LY30-mediated shift in Hsp27 oligomeric size (Figure 6a).
These results suggest that modulation of Hsp27 phosphorylation by LY30 directly influences the equilibrium between high- and low-molecular size Hsp27 oligomers in favor of the latter.

Hsp27 chaperone activity is inhibited by LY30. The capacity of Hsp27 to protect proteins from denaturation is proposed to be in part responsible for its anti-apoptotic activity. Hsp27 chaperone activity has been associated with the fraction of Hsp27 oligomers displaying high-molecular masses. On the basis of the effect of LY30 on Hsp27 oligomerization, we next asked whether LY30 had an effect on Hsp27 chaperone activity.

HeLa cells transiently expressing the renilla luciferase gene were treated as described in the experimental setup outlined in Supplementary Figure 5. As this assay measures the overall chaperone activity in the cell (i.e., the activity of all chaperone proteins), both specific overexpression and silencing of Hsp27 were used to determine the contribution of Hsp27 in the overall chaperone activity (Figure 7a). As seen in figure $7 \mathrm{~b}$, the heat shock was sufficient to denature luciferase efficiently (HSRO $+/-$ LY) and a $3 \mathrm{~h}$ recovery period was enough to observe a significant renaturation of the protein. Interestingly, Hsp27 overexpression was able to enhance protein renaturation by about $140 \%$, whereas Hsp27 silencing reduced it by $40 \%$ as compared with the control (Figure 7b), indicating that Hsp27 accounted for a significant part of the chaperone activity in the cell. More importantly, results show that LY30 treatment significantly reduced Hsp27 chaperone activity both in control and in Hsp27 overexpressing cells. 

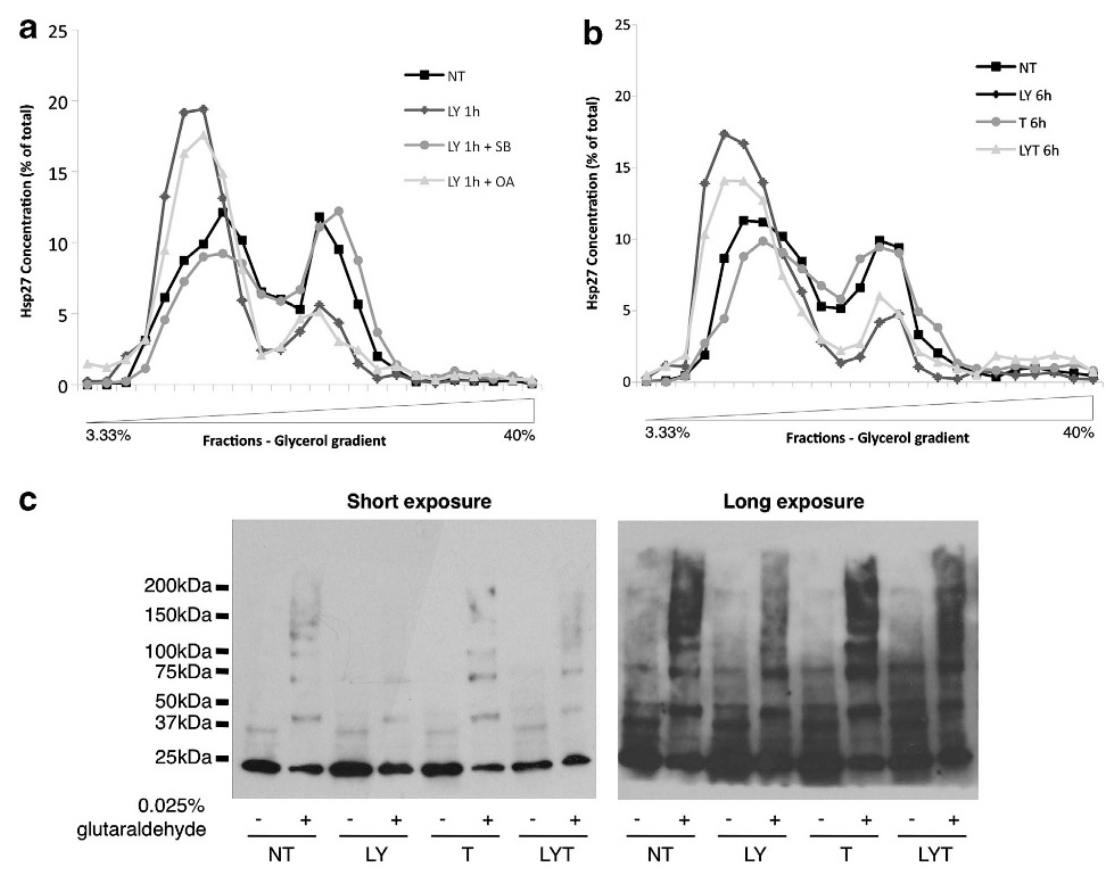

Figure 6 Effect of LY30 on Hsp27 oligomers size distribution. (a) HeLa cells were treated with $25 \mu \mathrm{M} \mathrm{LY} 30$ for $1 \mathrm{~h}$ in the presence or absence of $10 \mu \mathrm{M} \mathrm{SB203580}$ or $10 \mu \mathrm{M}$ okadaic acid (OA) (2 h preincubation) or (b) treated for $6 \mathrm{~h}$ with $20 \mathrm{ng} / \mathrm{ml}$ TRAlL with or without preincubation with $25 \mu \mathrm{M} \mathrm{LY} 30$. The cell extracts were fractionated by centrifugation on glycerol gradient. Each fraction was slot-blotted and Hsp27 was detected by immunoblotting using anti-HSP27. Immunoblots were then analyzed by densitometry. (c) Glutaraldehyde cross-linking of cell lysates from cells treated for $1 \mathrm{~h}$ with $20 \mathrm{ng} / \mathrm{ml}$ TRAlL with or without preincubation with $25 \mu \mathrm{M} L Y 30$

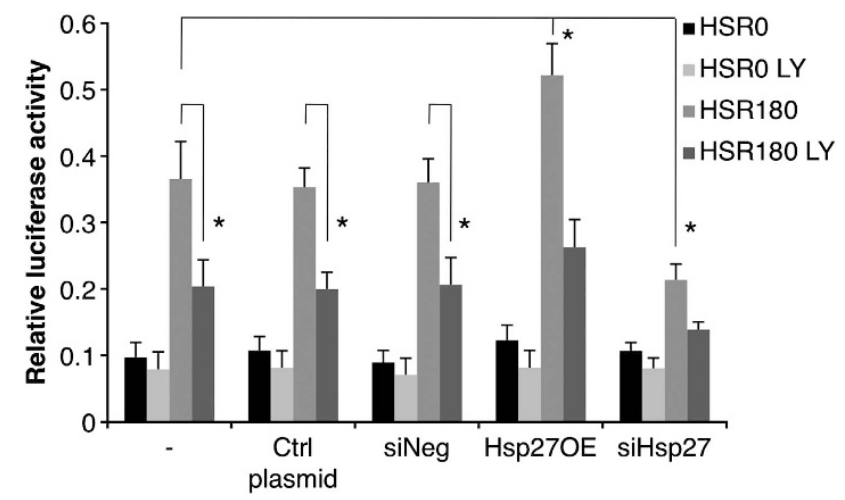

Figure 7 Effect of $\mathrm{LY} 30$ on in vivo chaperone activity. In vivo chaperone activity. A schematic experimental setup is provided in Supplementary Figure $5 .{ }^{\star} P<0.05$. HSR0: no recovery after heat-shock, HSR180: $3 \mathrm{~h}$ recovery after heat-shock

Taken together these results show that LY30 is able to induce profound biochemical changes to Hsp27 protein, thereby impairing its protective functions and relieving the breaks on apoptosis.

\section{Discussion}

In this study, we report a novel mechanism by which LY30 sensitizes tumor cells to TRAIL-mediated cell death that involves the alteration of Hsp27 protective functions. Our results show that, although $\mathrm{Hsp} 27$ expression remained unchanged upon exposure to LY30, its cellular localization was drastically changed. Indeed, we observed slow and sustained translocation of Hsp27 to the nucleus. Such translocation of Hsp27 has been extensively described in the literature in response to different type of cellular stress $^{24-26}$ and is usually rapid and transient. It is thought that such translocation allows Hsp27 to exert its protective functions in the nucleus for the duration of the cellular stress by sequestration of critical components of the translational and transcriptional machinery in order to prevent them to be damaged, ${ }^{27}$ and thus allows for a faster recovery upon return to physiological conditions. However, a sustained nuclear translocation of Hsp27 can be viewed as a nuclear sequestration of the protein. Indeed, the continual decrease in the cytosolic pool of Hsp27 is very likely to diminish the protection offered by this protein in the cytosol and might very well lead to a more efficient activation of caspases, in particular caspase3 and caspase- 9 .

As Hsp27 does not contain a nuclear localization signal, its transport to and from the nucleus is thought to be passive, through the nuclear pores, implying a small enough protein complex. In that regard, a study using Hsp27 fused to GFP reported that in some cell lines the fused protein was unable to enter the nucleus, ${ }^{28}$ indicating that $\mathrm{Hsp} 27$ needs to be in its monomeric or dimeric form in order to enter the nucleus. Hsp27 is present in the cell in the oligomeric form and oligomers of varying sizes are in a dynamic equilibrium regulated by Hsp27 phosphorylation. Hsp27 has been shown to be phosphorylated following exposure to several cellular stresses and drugs. ${ }^{29-35}$ Here, we show that Hsp27 was rapidly phosphorylated upon exposure to LY30 and that, unlike other reports its phosphorylation was sustained for up to $16 \mathrm{~h}$, which seems to indicate a constant upstream regulation. Stress-induced phosphorylation of $\mathrm{Hsp} 27$ is catalyzed mainly by p38 MAPK cascade and leads to the 
activation of MK2, a major kinase involved in Hsp27 phosphorylation. ${ }^{36-38}$ Indeed LY30 induced a rapid activation of p38 and MK2 and inhibition of p38 led to a marked decrease in MK2 and Hsp27 phosphorylation.

Hsp27 phosphorylation is also regulated by PP2A activity. ${ }^{39,40}$ We first confirmed that PP2A was functional and could affect Hsp27 phosphorylation before showing that PP2A was inhibited following exposure to LY30. A small, but significant, inhibition of PP2A (25\% inhibition) after $1 \mathrm{~h}$ of preincubation with LY30 suggests that Hsp27 rapid phosphorylation is likely mediated by rapid $\mathrm{p} 38 / \mathrm{MK} 2$ activation rather than fast inhibition of PP2A. The strong inhibition observed after $6 \mathrm{~h}$ of treatment $(60 \%)$ helps to explain the sustained phosphorylation of Hsp27. How LY30 activates p38 and inhibits PP2A remains to be answered. However, both p38 and PP2A have been shown to be sensitive to ROS, ${ }^{41-43}$ which is produced upon exposure to LY30 and can lead to p38 activation as shown by Shenoy et al. ${ }^{12}$ Moreover, PP2A can negatively regulate $\mathrm{p} 38$ and MK2. ${ }^{44,45}$ Hence, inhibition of PP2A by LY30 would enhance Hsp27 phosphorylation directly and/or indirectly. Interestingly, two studies by Song's et al. ${ }^{46,47}$ have recently shown that increased phosphorylation of Hsp27 and p38 was responsible for tilting the cell fate toward apoptosis during curcumin and TRAIL treatment via modulation of Akt survival pathway, hence strengthening our hypothesis that increased Hsp27 phosphorylation is important for TRAIL sensitization.

Having shown that LY30 induced changes in Hsp27 phosphorylation, we investigated the potential effects on Hsp27 oligomeric conformation. Corroborating earlier findings, we confirmed the existence of two distinct populations of oligomers in HeLa cells. ${ }^{48,49}$ The addition of LY30 induced a drastic and very rapid shift in Hsp27 oligomeric size in favor of the smaller oligomers. Furthermore, inhibition of PP2A led to a similar shift, whereas inhibition of p38 blocked the LY30mediated shift, thus providing a clear link between LY30mediated phosphorylation of Hsp27 and Hsp27 large oligomers dissociation. On a side note, LY30 did not lead to a complete and transient dissociation of Hsp27 oligomers into monomers and dimers, but rather to a mixture of small oligomers of varying sizes along with monomers and dimers, the composition of this mixture being sustained during the treatment. This might explain why the observed nuclear translocation of Hsp27 was slow and sustained rather that massive and transient.

Large Hsp27 oligomers provide Hsp27 with its chaperone activity. ${ }^{49}$ Interestingly, the overall activity is decreased by $40 \%$ when Hsp27 is silenced, whereas overexpression markedly enhances it (35\% increase). This indicates that Hsp27 is a major component of the chaperone machinery in HeLa cells. LY30 affects the overall chaperone activity in the cells and inhibits it by $40 \%$. Interestingly, even though the chaperone activity is enhanced in cells overexpressing Hsp27, LY30 leads to an inhibition comparable to that in control cells, which is indicative of a specific effect of LY30 toward Hsp27, independently of Hsp27 protein level. The inhibition of Hsp27 chaperone activity likely leaves the cells exposed to potentially toxic aggregates of denatured proteins. Interestingly, Hsp27 large oligomers not only serve to create large reservoir of misfolded proteins awaiting to be refolded or degraded but can also sequester functional, non-denatured proteins. On one hand, Hsp27 large oligomers have been shown to be responsible for the protection of key enzymes of the redox system, ${ }^{49,50}$ thereby favoring the formation of reduced glutathione and hence keeping ROS levels low. Thus, the dissociation of large oligomers observed following treatment with LY30 is likely to negate the beneficial effect of Hsp27 on cellular redox status. Moreover, the rapidity at which the large oligomers disappear (within $1 \mathrm{~h}$ ) could actually maximize the damage brought about by the early LY30mediated ROS burst. On the other hand, large oligomers have been demonstrated to be the active form for the general caspase-dependent anti-apoptotic effect of Hsp27. ${ }^{48}$ Likewise, this protective function could be greatly diminished as the large oligomeric population decreases.

Finally, the modulation of Hsp27 expression showed that Hsp27 represents an important apoptotic block in our cell system and that it not only has a significant role in LY30 sensitization to TRAIL but also in TRAIL resistance. These results are in line with findings from a recent study by Zhuang et al. ${ }^{51}$ showing that downregulation of $\mathrm{Hsp} 27$ could sensitize cells to TRAIL-induced apoptosis. Hence, the targeting of Hsp27, a protein with multiple protective roles, by LY30 translates into the weakening of tumor cells defenses and, in association with its other effects on TRAIL apical signaling, permits the generation and efficient transduction of an apoptotic signal.

\section{Conclusion}

In conclusion, we describe a novel mechanism where LY30 restores tumor cells sensitivity to TRAIL treatment by priming the cells via inhibition of Hsp27 protective function. This mechanism involves a sustained phosphorylation of Hsp27, through activation of the p38/MK2 pathway and the inhibition of PP2A, which in turn results in Hsp27 nuclear sequestration and inhibition of its chaperone activity due the loss of highmolecular weight Hsp27 oligomers. This study demonstrates the importance of targeting Hsp27 in order to alleviate TRAIL resistance in cancer cells and, taken together with our previous reports, describe LY30 as a truly pleiotropic small molecule with a strong potential to help build a better understanding on how to overcome drug resistance in cancer.

\section{Materials and Methods}

Reagents. The plasmid pcDNA-Hsp27 containing the full-length human hsp27 gene was a generous gift from Doctor Borelli (Department of Radiation Oncology, University of Arkansas Medical School, Little Rock, AR, USA). The pcDNA3.1 + vector was obtained from Invitrogen (Carlsbad, CA, USA). siRNAs for Hsp27 and the negative control siRNA were obtained from Dharmacon (Lafayette, CO, USA). Human recombinant TRAIL was purchased from Biomol International (Farmingdale, NY, USA), LY303511 from Alexis. Lactacystin, SB203580 and okadaic acid were purchased from Sigma-Aldrich (St. Louis, MO, USA).

Cell line and culture conditions. The human cervical cancer cell line HeLa was purchased from American Type Culture Collection (ATCC, Manassas, VA, USA). HeLa cells were cultured in Dulbecco's Modified Eagle's medium (Hyclone, Waltham, MA, USA) supplemented with 1\% L-glutamine, $1 \%$ streptomycin-penicillin and 10\% FBS (Gibco, Carlsbad, CA, USA).

Determination of cell viability and clonogenic growth. HeLa cells were treated as indicated and cell viability was assessed by the crystal violet staining method and trypan blue exclusion assay, whereas clonogenic growth was determined by crystal violet staining after 10-14 days of culture. 
Western blot analysis. Western blot analysis were performed as previously described $^{12}$ using antibodies against Hsp27 (Assay Designs/Stressgen, Farmingdale, NY, USA), phospho-Hsp27 (ser82), Hsp70, Hsp90, PARP, Smac (BD Pharmingen, San Jose, CA, USA), Actin, MAPKAP kinase 2 (MK2) (Cell Signaling Technology, Danvers, MA, USA), phospho-p38 (T180/Y182). Secondary antibodies conjugated to horseradish peroxidase (Pierce Biotechnology, Rockford, IL, USA) were used for detection and followed by enhanced chemiluminescence (Pierce Biotechnology) and autoradiography.

Densitometry. Densitometry measurements were done using the image analysis software Image $\mathrm{J}$ (http://rsbweb.nih.gov/ij)). When necessary, normalization was done against the loading control (actin).

DNA and siRNA transfections. For transient expression, HeLa cells $\left(10^{6}\right.$ cells per $\left.100 \mathrm{~mm}\right)$ were transfected with $10 \mu \mathrm{g}$ of pcDNA-Hsp27 or pcDNA3.1 + using the calcium phosphate transfection method. For knockdown of gene expression, $50 \mathrm{nM}$ siRNA (Hsp27 siRNA or negative control siRNA) was transfected according to the protocol provided by the manufacturer (Dharmacon). In each case, cells were cultured for $24 \mathrm{~h}$ before trypsinization and seeding for subsequent experiments.

PP2A activity assay. Following treatment, HeLa cells were lysed in a buffer containing $1 \mathrm{mM}$ EDTA, $150 \mathrm{mM} \mathrm{NaCl}, 50 \mathrm{mM}$ Tris- $\mathrm{HCl}(\mathrm{pH} 7.4)$ and $1 \%$ Triton X-100, supplemented with proteases inhibitors. The cell lysates were then sonicated and centrifuged for $15 \mathrm{~min}$ at $16000 \times \mathrm{g}$. PP2A activity in the supernatants was assayed according to the protocol provided by the manufacturer using (phosphatase kit V2460, Promega, Madison, WI, USA).

\section{Cellular fractionation}

Soluble/insoluble fractions: RIPA cell lysates were incubated on ice for 15 min with intermittent vortexing and freeze/thawed twice with another round of vortexing in-between. The lysates were then centrifuged at $16000 \times g$ for $15 \mathrm{~min}$. The supernatant was kept and the pellet was resuspended in $1 \times$ RIPA buffer.

Nuclear fractionation (4 and $8 \mathrm{~h}$ treatment): Following treatment, HeLa cells were harvested in PBS and nuclear fraction was performed as described by Cok et al. ${ }^{52}$

Alternative nuclear fractionation (16h treatment): Cells were resuspended in $400 \mu \mathrm{l}$ of buffer A (10 mM HEPES pH 7.9, $10 \mathrm{mM} \mathrm{KCl,} 100 \mathrm{mM}$ EDTA, $100 \mathrm{mM}$ EGTA) and incubated on ice for $15 \mathrm{~min}$. Then, $25 \mu \mathrm{l}$ of NP-40 was added to the cell suspension followed by vigorous vortexing for $10 \mathrm{~s}$. The cell suspension was then centrifuged at $16000 \times g$ for $30 \mathrm{~s}$ at $4^{\circ} \mathrm{C}$. The supernatant (cytosolic fraction) was stored at $-80^{\circ} \mathrm{C}$. The pellet (nuclear fraction) was resuspended in $50 \mu \mathrm{l}$ of ice-cold buffer C (20 mM HEPES pH 7.9, $400 \mathrm{mM} \mathrm{NaCl}, 1 \mathrm{mM}$ EDTA, $1 \mathrm{mM}$ EGTA) and incubated for $15 \mathrm{~min}$ at $4{ }^{\circ} \mathrm{C}$ before storage at $-80^{\circ} \mathrm{C}$.

Immunofluorescence analysis. Cells were fixed by adding $4 \%$ formaldehyde and quenched with $100 \mathrm{mM}$ glycine. $2 \%$ dodecyltrimethylammonium chloride was added to permeabilize the membrane and $50 \mathrm{mM} \mathrm{NH}_{4} \mathrm{Cl}_{2}$ to remove autofluorescence, before blocking with $5 \% \mathrm{BSA}$. Cells were probed with polyclonal rabbit anti-Hsp27 (Santa Cruz Biotechnology, Dallas, TX, USA). Immune complex formation was detected using an Alexa Fluor 568-conjugated secondary antibody (Molecular Probes/Invitrogen, Eugene, OR, USA). Nuclei were stained with Hoechst-33342 (Molecular Probes/Invitrogen). Pictures were taken with an Olympus BX53 microscope ( $\times 40$ magnification lens) and an Olympus DP72 camera. Olympus software cellSens Dimension was used to save and merge the images.

Hsp27 oligomerization analysis. The glycerol gradient centrifugation and the glutaraldehyde protocols were adapted from Lambert et $a l^{53}$ and performed using HeLa cells.

In vivo chaperone activity assay. The in vivo chaperone activity assay was adapted from Nollen et al. ${ }^{54} \mathrm{~A}$ schematic of the experimental setup is provided in the Supplementary Data section (Supplementary Figure 6).

Statistical analysis. All experiments were performed at least three times for statistical significance. Numerical data were expressed as mean \pm S.D. Statistical analysis was performed using the paired Student's $t$-test considering the variances unequal. $P$-values $<0.05$ were considered significant.

\section{Conflict of Interest}

The authors declare no conflict of interest.

Acknowledgements. We thank Ooi Zi Qi, Department of Physiology, National University of Singapore, for her assistance and contribution to this work. This work was supported by grants from the National Medical Research Council (NMRC), the Biomedical Research Council (BMRC), and the Ministry of Education (MOE) Tier 2 grant, Singapore to SP.

1. Wiley SR, Schooley K, Smolak PJ, Din WS, Huang CP, Nicholl JK et al. Identification and characterization of a new member of the TNF family that induces apoptosis. Immunity 1995; 3: 673-682.

2. Pitti RM, Marsters SA, Ruppert S, Donahue CJ, Moore A, Ashkenazi A. Induction of apoptosis by Apo-2 ligand, a new member of the tumor necrosis factor cytokine family. J Biol Chem 1996; 271: 12687-12690.

3. Walczak H, Miller RE, Ariail K, Gliniak B, Griffith TS, Kubin M et al. Tumoricidal activity of tumor necrosis factor-related apoptosis-inducing ligand in vivo. Nat Med 1999; 5: 157-163.

4. Ashkenazi A, Pai RC, Fong S, Leung S, Lawrence DA, Marsters SA et al. Safety and antitumor activity of recombinant soluble Apo2 ligand. J Clin Invest 1999; 104: 155-162.

5. Koschny R, Walczak H, Ganten TM. The promise of TRAIL-potential and risks of a novel anticancer therapy. J Mol Med 2007; 85: 923-935.

6. LeBlanc HN, Ashkenazi A. Apo2L/TRAIL and its death and decoy receptors. Cell Death Differ 2003; 10: 66-75.

7. Joy AM, Beaudry CE, Tran NL, Ponce FA, Holz DR, Demuth T et al. Migrating glioma cells activate the PI3-K pathway and display decreased susceptibility to apoptosis. J Cell Sci 2003; 116(Pt 21): 4409-4417.

8. Naka T, Sugamura K, Hylander BL, Widmer MB, Rustum YM, Repasky EA. Effects of tumor necrosis factor-related apoptosis-inducing ligand alone and in combination with chemotherapeutic agents on patients' colon tumors grown in SCID mice. Cancer Res 2002; 62: 5800-5806

9. Clayer M, Bouralexis S, Evdokiou A, Hay S, Atkins GJ, Findlay DM. Enhanced apoptosis of soft tissue sarcoma cells with chemotherapy: A potential new approach using TRAIL. J Orthop Surg (Hong Kong) 2001; 9: 19-22.

10. Zauli G, Sancilio S, Cataldi A, Sabatini N, Bosco D, Di Pietro R. PI-3K/Akt and NF-kappaB/ IkappaBalpha pathways are activated in Jurkat T cells in response to TRAIL treatment. $J$ Cell Physiol 2005; 202: 900-911.

11. Poh TW, Huang S, Hirpara JL, Pervaiz S. LY303511 amplifies TRAIL-induced apoptosis in tumor cells by enhancing DR5 oligomerization, DISC assembly, and mitochondrial permeabilization. Cell Death Differ 2007; 14: 1813-1825.

12. Shenoy K, Wu Y, Pervaiz S. LY303511 enhances TRAIL sensitivity of SHEP-1 neuroblastoma cells via hydrogen peroxide-mediated mitogen-activated protein kinase activation and up-regulation of death receptors. Cancer Res 2009; 69: 1941-1950.

13. Calderwood SK, Khaleque MA, Sawyer DB, Ciocca DR. Heat shock proteins in cancer: chaperones of tumorigenesis. Trends Biochem Sci 2006; 31: 164-172.

14. Ciocca DR, Calderwood SK. Heat shock proteins in cancer: diagnostic, prognostic, predictive, and treatment implications. Cell Stress Chaperones 2005; 10: 86-103.

15. Charette SJ, Lavoie JN, Lambert H, Landry J. Inhibition of Daxx-mediated apoptosis by heat shock protein 27. Mol Cell Biol 2000; 20: 7602-7612.

16. Bruey JM, Ducasse C, Bonniaud P, Ravagnan L, Susin SA, Diaz-Latoud C et al. Hsp27 negatively regulates cell death by interacting with cytochrome c. Nat Cell Biol 2000; 2 : $645-652$

17. Concannon CG, Orrenius S, Samali A. Hsp27 inhibits cytochrome c-mediated caspase activation by sequestering both pro-caspase-3 and cytochrome c. Gene Expr 2001; 9: 195-201.

18. Jakob U, Gaestel M, Engel K, Buchner J. Small heat shock proteins are molecular chaperones. J Biol Chem 1993; 268: 1517-1520.

19. Chou TC. Drug combination studies and their synergy quantification using the Chou-Talalay method. Cancer Res 2010; 70: 440-446.

20. Ohnishi K, Yasumoto J, Takahashi A, Ohnishi T. LY294002, an inhibitor of Pl-3K, enhances heat sensitivity independently of p53 status in human lung cancer cells. Int J Oncol 2006; 29: 249-253

21. Ramos AM, Fernandez C, Amran D, Sancho P, de Blas E, Aller P. Pharmacologic inhibitors of PI3K/Akt potentiate the apoptotic action of the antileukemic drug arsenic trioxide via glutathione depletion and increased peroxide accumulation in myeloid leukemia cells. Blood 2005; 105: 4013-4020.

22. Hansen RK, Oesterreich S, Lemieux P, Sarge KD, Fuqua SA. Quercetin inhibits heat shock protein induction but not heat shock factor DNA-binding in human breast carcinoma cells. Biochem Biophys Res Commun 1997; 239: 851-856.

23. Jakubowicz-Gil J, Paduch R, Gawron A, Kandefer-Szerszen M. The effect of heat shock, cisplatin, etoposide and quercetin on Hsp27 expression in human normal and tumour cells. Folia Histochem Cytobiol 2002; 40: 31-35.

24. Arrigo AP, Suhan JP, Welch WJ. Dynamic changes in the structure and intracellular locale of the mammalian low-molecular-weight heat shock protein. Mol Cell Biol 1988; 8: 5059-5071. 
25. McClaren M, Isseroff RR. Dynamic changes in intracellular localization and isoforms of the 27-kD stress protein in human keratinocytes. J Invest Dermatol 1994; 102: 375-381.

26. Wong JW, Shi B, Farboud B, McClaren M, Shibamoto T, Cross CE et al. Ultraviolet B-mediated phosphorylation of the small heat shock protein HSP27 in human keratinocytes. J Invest Dermatol 2000; 115: 427-434.

27. Cuesta R, Laroia G, Schneider RJ. Chaperone hsp27 inhibits translation during heat shock by binding elF4G and facilitating dissociation of cap-initiation complexes. Genes Dev 2000; 14: $1460-1470$.

28. Borrelli MJ, Bernock LJ, Landry J, Spitz DR, Weber LA, Hickey E et al. Stress protection by a fluorescent Hsp27 chimera that is independent of nuclear translocation or multimeric dissociation. Cell Stress Chaperones 2002; 7: 281-296.

29. Welch WJ. Phorbol ester, calcium ionophore, or serum added to quiescent rat embryo fibroblast cells all result in the elevated phosphorylation of two 28,000-dalton mammalian stress proteins. J Biol Chem 1985; 260: 3058-3062.

30. Arrigo AP, Welch WJ. Characterization and purification of the small 28,000-dalton mammalian heat shock protein. J Biol Chem 1987; 262: 15359-15369.

31. Kaur P, Welch WJ, Saklatvala J. Interleukin 1 and tumour necrosis factor increase phosphorylation of the small heat shock protein. Effects in fibroblasts, Hep G2 and U937 cells. FEBS Lett 1989; 258: 269-273.

32. Saklatvala J, Kaur P, Guesdon F. Phosphorylation of the small heat-shock protein is regulated by interleukin 1 , tumour necrosis factor, growth factors, bradykinin and ATP. Biochem J 1991; 277(Pt 3): 635-642.

33. Zhou M, Lambert H, Landry J. Transient activation of a distinct serine protein kinase is responsible for $27-\mathrm{kDa}$ heat shock protein phosphorylation in mitogen-stimulated and heat-shocked cells. J Biol Chem 1993; 268: 35-43.

34. Regazzi R, Eppenberger U, Fabbro D. The 27000 daltons stress proteins are phosphorylated by protein kinase $\mathrm{C}$ during the tumor promoter-mediated growth inhibition of human mammary carcinoma cells. Biochem Biophys Res Commun 1988; 152: $62-68$.

35. Arrigo AP. Tumor necrosis factor induces the rapid phosphorylation of the mammalian heat shock protein hsp28. Mol Cell Biol 1990; 10: 1276-1280.

36. Larsen JK, Yamboliev IA, Weber LA, Gerthoffer WT. Phosphorylation of the 27-kDa heat shock protein via p38 MAP kinase and MAPKAP kinase in smooth muscle. Am J Physiol 1997; 273(5 Pt 1): L930-L940.

37. Rouse J, Cohen P, Trigon S, Morange M, Alonso-Llamazares A, Zamanillo D et al. A novel kinase cascade triggered by stress and heat shock that stimulates MAPKAP kinase-2 and phosphorylation of the small heat shock proteins. Cell 1994; 78: 1027-1037.

38. Stokoe D, Engel K, Campbell DG, Cohen P, Gaestel M. Identification of MAPKAP kinase 2 as a major enzyme responsible for the phosphorylation of the small mammalian heat shock proteins. FEBS Lett 1992; 313: 307-313.

39. Berrou E, Bryckaert M. Recruitment of protein phosphatase $2 A$ to dorsal ruffles by plateletderived growth factor in smooth muscle cells: dephosphorylation of Hsp27. Exp Cell Res 2009; 315: 836-848.

40. Cairns J, Qin S, Philp R, Tan YH, Guy GR. Dephosphorylation of the small heat shock protein Hsp27 in vivo by protein phosphatase 2A. J Biol Chem 1994; 269: 9176-9183.

41. Rao RK, Clayton LW. Regulation of protein phosphatase $2 A$ by hydrogen peroxide and glutathionylation. Biochem Biophys Res Commun 2002; 293: 610-616.
42. Whisler RL, Goyette MA, Grants IS, Newhouse YG. Sublethal levels of oxidant stress stimulate multiple serine/threonine kinases and suppress protein phosphatases in Jurkat $T$ cells. Arch Biochem Biophys 1995; 319: 23-35.

43. Kulisz A, Chen N, Chandel NS, Shao Z, Schumacker PT, Mitochondrial ROS. initiate phosphorylation of p38 MAP kinase during hypoxia in cardiomyocytes. Am J Physiol Lung Cell Mol Physiol 2002; 282: L1324-L1329.

44. Stokoe D, Campbell DG, Nakielny S, Hidaka H, Leevers SJ, Marshall C et al. MAPKAP kinase-2; a novel protein kinase activated by mitogen-activated protein kinase. EMBO J 1992; 11: 3985-3994.

45. Alvarado-Kristensson M, Andersson T. Protein phosphatase $2 A$ regulates apoptosis in neutrophils by dephosphorylating both p38 MAPK and its substrate caspase 3 . J Biol Chem 2005; 280: 6238-6244.

46. Kim J, Kang D, Sun BK, Kim JH, Song JJ. TRAIL/MEKK4/p38/HSP27/Akt survival network is biphasically modulated by the Src/CIN85/c-Cbl complex. Cell Signal 2013; 25: 372-379.

47. Kim J, Kim SY, Kang S, Yoon HR, Sun BK, Kang D et al. HSP27 modulates survival signaling networks in cells treated with curcumin and TRAIL. Cell Signal 2012; 24: 1444-1452.

48. Bruey JM, Paul C, Fromentin A, Hilpert S, Arrigo AP, Solary E et al. Differential regulation of HSP27 oligomerization in tumor cells grown in vitro and in vivo. Oncogene 2000; 19 4855-4863.

49. Rogalla T, Ehrnsperger M, Preville X, Kotlyarov A, Lutsch G, Ducasse C et al. Regulation of Hsp27 oligomerization, chaperone function, and protective activity against oxidative stress/tumor necrosis factor alpha by phosphorylation. J Biol Chem 1999; 274 18947-18956.

50. Mehlen P, Kretz-Remy C, Preville X, Arrigo AP. Human hsp27, Drosophila hsp27 and human alphaB-crystallin expression-mediated increase in glutathione is essential for the protective activity of these proteins against TNFalpha-induced cell death. EMBO J 1996; 15: $2695-2706$.

51. Zhuang H, Jiang W, Cheng W, Qian K, Dong W, Cao L et al. Down-regulation of HSP27 sensitizes TRAIL-resistant tumor cell to TRAIL-induced apoptosis. Lung Cancer 2010; 68 27-38.

52. Cok SJ, Acton SJ, Morrison AR. The proximal region of the 3'-untranslated region of cyclooxygenase-2 is recognized by a multimeric protein complex containing $\mathrm{HuR}$, TIA-1, TIAR, and the heterogeneous nuclear ribonucleoprotein U. J Biol Chem 2003; 278 : 36157-36162.

53. Lambert H, Charette SJ, Bernier AF, Guimond A, Landry J. HSP27 multimerization mediated by phosphorylation-sensitive intermolecular interactions at the amino terminus. J Biol Chem 1999; 274: 9378-9385.

54. Nollen EA, Brunsting JF, Roelofsen $\mathrm{H}$, Weber LA, Kampinga HH. In vivo chaperone activity of heat shock protein 70 and thermotolerance. Mol Cell Biol 1999; 19: 2069-2079.

(c) (i) $\odot$ Cell Death and Disease is an open-access journal (a) licensed under a Creative Commons Attribution-NonCommercialNoDerivs 3.0 Unported License. To view a copy of this license, visit http://creativecommons.org/licenses/by-nc-nd/3.0/

Supplementary Information accompanies this paper on Cell Death and Disease website (http://www.nature.com/cddis) 